\title{
Retinoic Acid Receptor $\beta$ Controls Development of Striatonigral Projection Neurons through FGF-Dependent and Meis1-Dependent Mechanisms
}

\author{
Monika Rataj-Baniowska, ${ }^{1,2,3,4,5}$ Anna Niewiadomska-Cimicka, ${ }^{1,2,3,4,5}$ @Marie Paschaki, ${ }^{1,2,3,4,5}$ \\ Monika Szyszka-Niagolov, ${ }^{1,2,3,4,5}$ @Laura Carramolino, ${ }^{6}$ Miguel Torres, ${ }^{6}$ Pascal Dollé, ${ }^{1,2,3,4,5}$ and $\odot$ Wojciech Krężel ${ }^{1,2,3,4,5}$ \\ ${ }^{1}$ Developmental Biology and Stem Cells Department, Institut de Génétique et de Biologie Moléculaire et Cellulaire, ${ }^{2}$ Institut de la Santé et de la Recherche \\ Médicale, U964, ${ }^{3}$ Centre National de la Recherche Scientifique, Unité Mixte de Recherche 7104, ${ }^{4}$ Fédération de Médecine Translationnelle de Strasbourg, \\ and ${ }^{5}$ Université de Strasbourg, 67404 Illkirch, France, and ${ }^{6}$ Centro Nacional de Investigaciones Cardiovasculares, CNIC, Madrid 28029, Spain
}

The mammalian striatum controls sensorimotor and psychoaffective functions through coordinated activities of its two striatonigral and striatopallidal output pathways. Here we show that retinoic acid receptor $\beta(\operatorname{RAR} \beta)$ controls development of a subpopulation of GABAergic, Gad65-positive striatonigral projection neurons. In Rarb $^{-1-}$ knock-out mice, concomitant reduction of Gad65, dopamine receptor D1 (Drd1), and substance P expression at different phases of prenatal development was associated with reduced number of Drd1-positive cells at birth, in contrast to normal numbers of striatopallidal projection neurons expressing dopamine receptor D2. Fate mapping using BrdU pulse-chase experiments revealed that such deficits may originate from compromised proliferation of late-born striosomal neurons and lead to decreased number of Drd1-positive cells retaining BrdU in postnatal day (P) $0 \mathrm{Rarb}^{-1-}$ striatum. Reduced expression of $\mathrm{Fg} 33$ in the subventricular zone of the lateral ganglionic eminence (LGE) at embryonic day 13.5 may underlie such deficits by inducing premature differentiation of neuronal progenitors, as illustrated by reduced expression of the proneural gene Ascl1 (Mash1) and increased expression of Meis1, a marker of postmitotic LGE neurons. In agreement with a critical role of FGF3 in this control, reduced number of Ascll-expressing neural progenitors, and a concomitant increase of Meis1-expressing cells, were observed in primary cell cultures of $\mathrm{Rarb}^{-1-}$ LGE. This defect was normalized by addition of fibroblast growth factor (FGF). Such data point to role of Meis1 in striatal development, also supported by reduced neuronal differentiation in the LGE of Meisl ${ }^{-/}$embryos. Our data unveil a novel mechanism of development of striatonigral projection neurons involving retinoic acid and FGF, two signals required for positioning the boundaries of Meis1-expressing cells.

Key words: dopamine receptors; FGF; MEIS1; neurogenesis; retinoic acid receptors; striatum

Significance Statement

We provide the first evidence that retinoic acid signaling controls development of striatonigral projection neurons, which constitute one of the two major output pathways of the striatum. Our data point to retinoic acid receptor $\beta(\operatorname{RAR} \beta)$ as novel determinant of striatonigral pathway development, and indicate that such activities of RAR $\beta$ are mediated by abnormal FGF3 and Meis 1 signaling, but do not involve Isl1, Ctip2, or Ebf1, the only factors known so far to control development of these neurons. Furthermore, present data support possibility that lateral ganglionic eminence development is controlled by gradients of fibroblast growth factor and RA signaling.

\section{Introduction}

The striatum, the primary input structure of basal ganglia, derives from the embryonic lateral ganglionic eminence (LGE;

Received April 1, 2015; revised Aug. 17, 2015; accepted Sept. 8, 2015.

Author contributions:W.K. designed research; M.R.-B., A.N.-C., M.P., M.S.-N., L.C., and W.K. performed research; L.C., M.T., and P.D. contributed unpublished reagents/analytic tools; M.R.-B., A.N.-C., M.P., M.S.-N., and W.K. analyzed data; M.R.-B. and W.K. wrote the paper.

This work was supported by funds from Centre National de la Recherche Scientifique, Institut de la Santé et de la Recherche Médicale, Université de Strasbourg; and grants from the Agence Nationale de la Recherche (Programme Neurosciences 2008 and Programme Blanc 2012) and Fondation pour la Recherche Médicale (FRM;
Molero et al., 2009). Medium spiny projection neurons (MSNs) constitute a vast majority $(90-95 \%)$ of striatal neurons and are

DEQ20071210526). L.C. and M.T. were supported by MINECO Grant BFU2012-31086. M.R.-B. was supported by PhD fellowships from FRM, France Parkinson, and Fondation de France.We thank Valerie Fraulob, Brigitte Schuhbaur, and Marion Ciancia for technical assistance.

The authors declare no competing financial interests.

Correspondence should be addressed to Wojciech Krężel at the above address. E-mail: krezel@igbmc.fr.

M. Paschaki's present address: CGphiMCUMR 5534, Université Lyon-1, 16 rue Dubois, 69622 Villeurbanne, France

DOI:10.1523/JNEUROSCI.1278-15.2015

Copyright $\odot 2015$ the authors $\quad 0270-6474 / 15 / 3514467-09 \$ 15.00 / 0$ 
formed in the proliferative zones of the LGE including ventricular zone (VZ) and subventricular zone (SVZ). Newly formed progenitor cells migrate radially to the adjacent mantle zone (MZ), where they segregate into two discrete compartments: the striosomes (also called patches) and surrounding matrix. In mice, formation of striosomes between embryonic day (E) 11 and E14 precedes formation of matrix at E16-P0 and is associated with different afferent connections of these domains (van der Kooy and Fishell, 1987). Although MSNs are intermixed in both compartments, they can be divided according to targets of their axonal projections into striatonigral and striatopallidal projection neurons, which form the main striatal output pathway and provide, respectively, direct or indirect connections with substantia nigra. Whereas both types of projection neurons are GABAergic and express two forms of glutamic acid decarboxylases (Gad67 and/or Gad65), they also display a number of molecular differences, including expression of dopamine D1 receptor (Drd1) and substance $\mathrm{P}(\mathrm{SP})$ in striatonigral neurons and dopamine $\mathrm{D} 2$ receptor (Drd2), proenkephaline (Penk), or adenosine A2A receptor (Adora2a) in striatopallidal neurons. Coordinated activities of these pathways are important for control of motor, affective, and cognitive functions, which are underlined by a wide range of behavioral abnormalities associated with their imbalanced activity in degenerative diseases, such as Huntington disease and Parkinson disease, in drug abuse, or in depression (Crittenden and Graybiel, 2011). Despite a vast knowledge about molecular and functional differences between striatonigral and striatopallidal pathways, developmental mechanisms underlying generation of these two types of projection neurons are only beginning to be elucidated. Recent reports have focused on Isl1 (Ehrman et al., 2013; Lu et al., 2014), Ctip2 (Arlotta et al., 2008), or Ebf1 (Lobo et al., 2008), which through different mechanisms preferentially control development of the striatonigral pathway. In particular, Isl1 was shown to promote development, but also maintenance, of striatonigral neurons (Lu et al., 2014). Mechanisms coordinating expression and activities of these different transcription factors in LGE are not known, although the two potential candidates could include fibroblast growth factors (FGFs) and retinoic acid (RA), both acting as diffusible molecules critical for brain morphogenesis, cell-type specification, and differentiation. FGFs are known to control expansion of neural progenitors in different brain regions, including the telencephalon (Diez del Corral et al., 2003; Storm et al., 2003, 2006; Gutin et al., 2006; Theil et al., 2008), and signaling through FGF receptors $1-3$ has been proposed to mediate organizer activity in developing LGE (Paek et al., 2009). In agreement with the high differentiating potential of

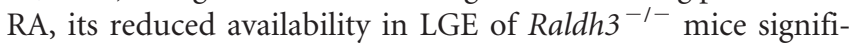
cantly decreased differentiation of GABAergic projection neurons (Chatzi et al., 2011). The RA signal could be mediated through two RA receptors (RARs), RAR $\alpha$, which is expressed in the SVZ region, or RAR $\beta$, which is expressed in the SVZ region as well as at high levels in the MZ (Ruberte et al., 1993). RAR $\beta$ was shown to control development of striosomal compartments (Liao et al., 2008), but its contribution to development of striatal projection neurons has not been assessed. Here we show that genetic ablation of RAR $\beta$ leads to selective reduction of striatonigral projection neurons. Premature neuronal differentiation reflected by ectopic expression of the postmitotic marker Meis1 in VZ and SVZ neurons, at the expense of progenitor cells expressing the proneural gene Ascl1 (Mash1), may directly underlie such deficits and result from reduced FGF3 signaling, one of the direct transcriptional targets of RA signaling.

\section{Materials and Methods}

Animals and tissue preparation. Null mutant mice and embryos for RAR $\beta$ $\left(\right.$ Rarb $\left.^{-1-}\right)$ and null mutant embryos for Meis1 $\left(\right.$ Meis $\left.1^{-/-}\right)$, and their respective wild-type (WT) littermate controls, were generated on a mixed genetic background (129/SvEmj; C57BL/6J) from heterozygous crosses as previously described (Ghyselinck et al., 1997; Azcoitia et al., 2005). Meanwhile, WT and Rarb ${ }^{-1-}$ embryos used for primary cultures of LGE cells were generated from homozygous crosses (WT $\times$ WT and $\mathrm{Rarb}^{-1-} \times \mathrm{Rarb}^{-1-}$ ) of mice with the same genetic background. The day of the vaginal plug following overnight mating was considered as $0.5 \mathrm{~d}$ postcoitum (E0.5). Genotypes of all animals were determined using appropriate PCRs (genotyping protocols available upon request).

For immunohistochemical and in situ hybridization (ISH) analyses, embryonic or adult brains were dissected on ice, fixed with $4 \%$ paraformaldehyde (PFA) in phosphate-buffered saline (PBS), cryoprotected in $20 \%$ sucrose solution in PBS for $24 \mathrm{~h}$, embedded in Shandon Cryomatrix (Thermo Scientific), frozen, and stored at $-80^{\circ} \mathrm{C}$. Coronal cryosections (14 $\mu \mathrm{m}$ thick) were collected onto Superfrost plus slides (Menzel-Glaser) and stored at $-80^{\circ} \mathrm{C}$ until analysis.

Short-term and long-term BrdU tracing. Pregnant females were injected intraperitoneally with $100 \mathrm{mg} / \mathrm{kg} \operatorname{BrdU}$ (Sigma-Aldrich) at E13 and killed at different time points to conduct the necessary experiment. Quantification of the population of proliferating cells was performed as described previously (Martynoga et al., 2005; Tucker et al., 2010). Briefly, for birthdating analyses, pregnant females were injected with BrdU at E13.0 and the embryos or brains were collected at E13.5, E18.5, or at birth (P0), and processed for immunohistochemical analyses.

Primary cell culture. To obtain necessary material for primary LGE cultures and to ensure consistant experimental conditions, we pooled LGEs from 4-5 embryos (E13.5) of a given genotype to constitute one experimental sample, and $\geq 4$ samples were analyzed for each genotype in each experimental condition. Dissected LGEs were incubated in nonenzymatic dissociation buffer (Invitrogen, $13150-016$ ) at $37^{\circ} \mathrm{C}$ for $15 \mathrm{~min}$ and subsequently triturated using fire-polished Pasteur glass pipettes to obtain single-cell suspension. For cell-pair assays, cells were plated at a concentration of $60-80 \times 10^{3}$ cells $/ \mathrm{ml}$ on $12 \mathrm{~mm}$ glass coverslips precoated with laminin (BD Bioscience, 354232) and cultured for $24 \mathrm{~h}$ in a humidified incubator at $37^{\circ} \mathrm{C}$ with $5 \% \mathrm{CO}_{2}$. Cells were cultured in neurobasal medium (Invitrogen, 21103-049) with N2 supplement (Invitrogen, 17502-048) or in medium enriched with trophic factors containing B27 (Invitrogen, 12587-010) and 40 ng/ml FGF2 (human; Peprotech).

Immunohistochemistry and immunocytochemistry. Slides with fixed tissue sections or cultured cells were blocked with normal goat serum or fetal calf serum and incubated with the following primary antibodies: rat BrdU (1:10; Serotec, OBT0030S); rabbit Ki67 (1:200; Novocastra, NCLKi67p); rabbit Meis1 (1:400; Azcoitia et al., 2005); mouse Ascl1/Mash1 (1:200; BD PharMingen, 556604); rabbit Ascl1/Mash1 (1:10,000; a gift from Jane E. Johnson, University of Texas Southwestern Medical Center); mouse Is11/2 (1:100; 39.4D5 from Hybridoma Bank); rabbit Foxp1 (1:500; Abcam, AB16645); activated caspase 3 (1:200; BioVision, 3015100). Heat-induced antigen retrieval was used for immunodetection of BrdU. Primary antibodies were visualized using secondary antibodies from donkey conjugated with Alexa 488 or Alexa 555 (1:1000; Fisher Scientific), whereas cell nuclei were identified using DAPI (1:2000).

ISH and quantitative real-time PCR. Chromogenic ISHs were performed with digoxigenin-labeled riboprobes targeting gene-specific regions described previously: Rara and Rarb (Dollé et al., 1990); Gad65 (Erlander et al., 1991), Ascl1 (Mash1, full-length cDNA), Ikzf1 (fulllength cDNA), Meis 1 (nucleotides 415-475 region of cDNA); Dlk1 (fulllength cDNA, gift from J. Lewis); Fgf3 (532 bp 3' cDNA region; gift from D. Wilkinson); Foxp1 (nucleotides 350-700 region of cDNA); Isl1 (nucleotides 380-513 region of cDNA), Ctip2 (full-length cDNA), Pdyn (1.7kb 3' region of cDNA), Chmr4 (full-length cDNA). The expression of each gene was analyzed in $n=4-6 \mathrm{WT}$ and $n=4-6 \mathrm{Rarb}^{-1-}$ biologically independent embryos or newborn mice (as specified in the text) using several sections of the striatum at corresponding anteroposterior positions and separated by $70 \mu \mathrm{m}$. Hybridization conditions were as described previously (Krezel et al., 1998). Detailed protocols for probe 
synthesis and ISH are available on the website http://www.empress.har. mrc.ac.uk/browser: gene expression section. For BrdU detection after ISH, antigen retrieval was performed before ISH, whereas immunofluorescent detection of BrdU was performed, using anti-BrdU monoclonal antibody followed by Alexa 488 secondary antibody, simultaneously with immunodetection of dig-labeled Drd1 or Drd2 antisense probes. For fluorescent ISH, we used frozen brain section from $\mathrm{P} 0$ pups that were deep anesthetized and perfused with $4 \%$ PFA. Detection of probes was done using peroxidaseconjugated sheep anti-dig antibody (reference \#11207733910, Roche) and revealed with TSA Plus Cyanine 3 System (PerkinElmer). Quantitative realtime PCR (qRT-PCR) was performed on dissected E13.5, E16.5, and E18.5 LGE samples ( $n=5 \mathrm{WT}$ and $5 \mathrm{Rarb}^{-/-}$for each stage) and normalized for expression of a housekeeping gene $36 B 4$ as previously described (Krzyzosiak et al., 2010). PCRs were performed in duplicate or triplicate with good reproducibility rate (error, $<10 \%$ ). Forward and reverse primers used for these analyses were respectively as follows: Adora2a: CAGAG TTCCATCTTCAGCCTC, CACCCAGCAAATCGCAATG; Ascll: TTCT CCGGTCTCGTCCTACTC, CCAGTTGGTAAAGTCCAGCAG; Ctip2: T GCGTCTGTACAGCGTGAC, ACTTCGCAGACACAGGTTAGG; Dlk1:GA AAGGACTGCCAGCACAAG, CACAGAAGTTGCCTGAGAAGC; Drd1: AAGATGCCGAGGATGACAAC, CCCTCTCCAAAGCTGAGATG; Drd2: TCGCCATTGTCTGGGTCCTG, TGCCCTTGAGTGGTGTCTTC; Ebf1: AG GTACGCCCTCTTATCTGG, GACCAGCATGGTACCGAATA; Gad65: GGGATGTCAACTACGCGTTTC, GAGTGGGCCTTTCTCCATCA; Gad67: GGGTTCCAGATAGCCCTGAGCGA, TGGCCTTGTCCCCTTGAGG CT; Fgf3: GATTACTGCGGTGGAAGTGG, GCGTTGTAGTGATC CGAAGC; Isl1: CAGCAACCCAACGACAAAAC, GTCACTCAGTACTT TCCAGGG; Meis1: GGTGTTCGCCAAACAGATT, GGTGGCAGAAATT GTCACAT; Penk, AAAATCTGGGAGACCTGCAA, TCTTCTGGCTCCA TGGGATA; Raldh3: AGTGTGGAGTTCGCCAAGAAGAGG, AGACCG TGGGTTTGATGAACAGCC; SP (Tac1): AGGCTCTTTATGGACAT GGC, TCTTTCGTAGTTCTGCATCGC.

Cell counts and statistical analyses. All analyses and imaging were performed using Leica M420 or DMLB/DM4000B microscopes equipped with Photometrics digital cameras and the CoolSnap imaging software (Roger Scientific). The cells were counted manually using ImageJ software (Rasband, http://rsb.info.nih.gov/ij/) and automatically using a software developed in the laboratory (M. Baniowski, unpublished). Both methods gave similar results. For the primary cell cultures, DAPI-positive cells were scored to quantify global cell number. Adjacent DAPI-positive cells were considered as divided cell pairs. BrdU-positive, Drd1-positive, or Drd2-positive cells were quantified in both hemispheres from LGE and striatum using 3-4 sections separated by $70 \mu \mathrm{m}$ for E13.5 or by $100 \mu \mathrm{m}$ for E18.5 embryos and P0 mice (only the latter were used for Drd1 and Drd2 cell counts). Two independent experimenters counted cells from the same areas.

Statistical analyses were performed using Student's $t$ test to compare WT and Rarb ${ }^{-1-}$ samples (e.g., on qRT-PCR data). For analyses of FGF effects in WT and Rarb $^{-/-}$primary LGE cell cultures, two-way ANOVA was used with genotype and treatment as independent variables. ANOVA analyses were followed by Student's $t$ test post hoc comparisons (StatView software).

\section{Results}

\section{Proliferation deficits result in reduced number of} striatonigral projection neurons in the developing striatum of perinatal Rarb $^{-I-}$ mice

Consistent with a previous report (Liao et al., 2008), we observed that a single injection of BrdU during the early wave of neurogenesis at E13.0 marked fewer cells in the SVZ of the Rarb ${ }^{-1-}$ LGE than in the SVZ of WT LGE when assayed $2 \mathrm{~h}$ after injection (Rarb ${ }^{-1-}$ attained $82 \pm 5 \%$ of WT cell counts; Fig. $1 A, A^{\prime}$ ), and such difference was even stronger when counting BrdU + cells in the MZ of the Rarb ${ }^{-1-}$ LGE at E18.5 (61 $\pm 5 \%$ of WT cells; Fig. $\left.1 B, B^{\prime}\right)$ or P0 ( $69 \pm 4 \%$ of WT cells; Fig. $\left.1 C 2, D 2, I\right)$. To test whether reduced cell proliferation at E13.0 results in reduced numbers of projection neurons, we quantified the numbers of $D r d 1+$ and $D r d 2+$ neurons in the striatum of newborn mice
(P0) by fluorescence ISH, and determined whether they retained BrdU injected at E13.0 (Fig. 1C1-D4). We observed a significant $(\sim 50 \%)$ reduction of $D r d 1$ and BrdU double-labeled cells, which was associated with an overall $23 \pm 8 \%$ reduction of $\operatorname{Drd1} 1+$ neurons in $\mathrm{Rarb}^{-1-}$ striatum (Fig. 1I), indicating a deficit in the number of striatonigral projection neurons generated during this period of neurogenesis. In contrast, the number of Drd2-positive neurons including those that accumulated BrdU injected at E13.0 was not different between WT and Rarb ${ }^{-1-}$ striatum (Fig. 1I). Reduction of Drd1-positive neurons, evident also at E18.5 (Fig. $\left.1 E, E^{\prime}\right)$, was restricted to striosomal compartments, as prodynorphin, a marker of striosomal Drd1 neurons, was strongly diminished in the anterior part of $\mathrm{Rarb}^{-1-}$ striatum (Fig $1 F, F^{\prime}$ ), whereas expression of Chrm4, a marker of matrix Drd1 neurons was not affected (Fig $1 G, G^{\prime}$ ). In agreement with a previous report (Liao et al., 2008), striosomal compartmentalization of prodynorphin expression was affected mostly in anterior, but not in posterior, striatum. Expression of $\mathrm{Drd} 2$ was not reduced throughout E18.5 $\mathrm{Rarb}^{-1-}$ striatum (Fig. $1 \mathrm{H}, \mathrm{H}^{\prime}$ ). In concordance with reduced number of striatonigral projection neurons, levels of Drd1 and SP, an additional marker of striatonigral projection neurons, were significantly lower in $\mathrm{Rarb}^{-1-}$ LGE at E18.5 as assessed by qRT-PCR (Fig. $1 J$ ). There was no effect of RAR $\beta$ ablation on the expression of striatopallidal markers including Drd2, Penk1, or Adora2a (Fig. 1I), supporting the idea that this pathway is not affected in Rarb ${ }^{-1-}$ mice. Interestingly, whereas expression of Gad67 was not affected, the expression of Gad65, preferentially associated with functions of striatonigral projection neurons (Laprade and Soghomonian, 1997), was decreased, further suggesting pathway-specific deficits (Fig. $1 J$ ).

\section{Ectopic expression of Isl1 and Meis1 in VZ and SVZ indicates premature differentiation of neural progenitors in the Rarb $^{-1-}$ LGE}

To identify mechanisms underlying deficient generation of striatonigral projection neurons, we analyzed the expression of Isl1, Ctip2, and Ebf1, known to contribute to specification of these cells. The mRNA levels and distribution of these determinants were not affected at E13.5 with exception of a weak decrease of Ctip2 expression (Fig. $2 A-B^{\prime}, I$ ). However, cells expressing high levels of Isl1 protein were found in the SVZ (Fig. 2, compare G1-G3 for WT, $G 1^{\prime}-G 3^{\prime}$ for $\mathrm{Rarb}^{-/-}$) and sporadically in the VZ of $\mathrm{Rarb}^{-/-}$LGE, where their expression was restricted to Ascl1+ neural progenitor cells (Fig. 2G3', arrows). Such distribution of Isl1, normally expressed at high levels in postmitotic differentiating neurons of the MZ of the LGE, suggested that premature differentiation of neural progenitor cells may underlie the reduced proliferation index we observed in the Rarb ${ }^{-1-}$ striatum. To test this hypothesis, we investigated expression of Meis1, a marker of postmitotic differentiated neurons in the striatum. Using ISH (Fig. 2C, $C^{\prime}$ ) and qRT-PCR (Fig. 2I), we found a significant increase of Meis 1 expression, distributed in patchy zones of ectopic expression in the VZ (Fig. $2 C, C^{\prime}$ ) in 4 of 5 analyzed embryos, which was accompanied by reduction of Ascll expression in Rarb $^{-1-}$ VZ of the LGE (Fig. 2D, $\left.D^{\prime}, \mathrm{I}\right)$. Consistent with Meis1 overexpression being functionally relevant, expression of Dlk1, a direct transcriptional target of Meis1 (Argiropoulos et al., 2008), known to promote neurogenesis of human and mouse neural progenitors (Surmacz et al., 2012), was also increased in Rarb $^{-1-}$ LGE and displayed ectopic expression in the VZ region (Fig. 2E, $E^{\prime}, I$ ) with high penetrance (four of five analyzed embryos). Immunohistological analyses confirmed increased Meis1 expression and revealed the presence of Meis1-positive cells in 
ectopic, patchy-like regions of the $\mathrm{VZ}$ of mutant embryos illustrated in Figure 2 (compare $H 1-H 3, H 1^{\prime}-H 3^{\prime}$ ). In contrast, Ascl1-expressing cells were significantly reduced in $\mathrm{Rarb}^{-/-} \mathrm{VZ}(72 \pm 10 \%$ of WT; $p<0.05)$ and some of them also coexpressed Meis1.

Along with premature neuronal differentiation associated with enhanced and ectopic expression of Meis1 in the $\mathrm{Rarb}^{-1-}$ striatum at E13.5, we identified by qRT-PCR reduced expression of Foxp1 $(77 \pm 6 \%$ of WT, $p<0.05)$ and Ikzfl (37 \pm $15 \%$ of WT, $p<0.05$ ), distinct markers of neuronal differentiation in the LGE. This result was intriguing since Foxp1 is a broadspectrum marker of projection neurons (Tamura et al., 2004) and Ikzf1 preferentially controls development of striatopallidal projection neurons (Martín-Ibáñez et al., 2010), whereas deficit detected at E18.5 in the Rarb $^{-I-}$ LGE was restricted to striatonigral projection neurons (Fig. $1 F$ ). We therefore tested the hypothesis that more severe deficits in differentiation of projection neurons or their subtypes might be present at earlier stages of development than E18.5. In support of increased severity of deficits, expression of Gad65 (60.9\% of WT; $p<$ 0.007 ) and $\operatorname{Gad} 67$ (63.7\% of WT, $p<0.05)$, markers of differentiated GABAergic projection neurons, was strongly reduced at E16.5. However, these changes reflected compromised differentiation of striatonigral projection neurons as there was reduced expression of $\operatorname{Drd1}$ ( $51 \%$ of WT, $p<$ $0.04)$ and $S P(33.7 \%$ of WT, $p=0.05)$, contrasting with normal expression of $\operatorname{Drd} 2$ (104\% of WT; ns) and Penk (100.2\% of WT; not significant) in the E16.5 $\mathrm{Rarb}^{-/-}$LGE. In agreement with a previous report (Liao et al., 2008), we did not detect any differences in apoptosis using TUNEL in $\mathrm{Rarb}^{-1-}$ LGE at E13.5, E16.5, or E18.5.

Premature differentiation is associated with reduced FGF signaling in the

Rarb $^{-1-}$ LGE

The effect of loss of function of $\operatorname{RAR} \beta$ expressed in the MZ/SVZ region of the LGE (Liao et al., 2003) on the proliferation of progenitor cells located in the VZ was intriguing. To address the mechanisms of such long-range $\operatorname{RAR} \beta$ functions, we asked whether abnormal signaling through diffusible factors controlling maintenance and differentiation of neural progenitors could be altered in the $\mathrm{Rarb}^{-1-}$ LGE. We focused these analyses on RA and FGFs acting in the SVZ and/or VZ through their respective receptors, RAR $\alpha$ and FGFR1-2. Since expression of Raldh 3 and Rara were not affected (Fig. 2I), we focused further analyses on FGFs, known as important inhibitors of terminal differentiation, including FGF3 and FGF8 controlling early development of the LGE (Theil et al., 2008). At E13.5, Fgf8 transcripts were detected only in the developing
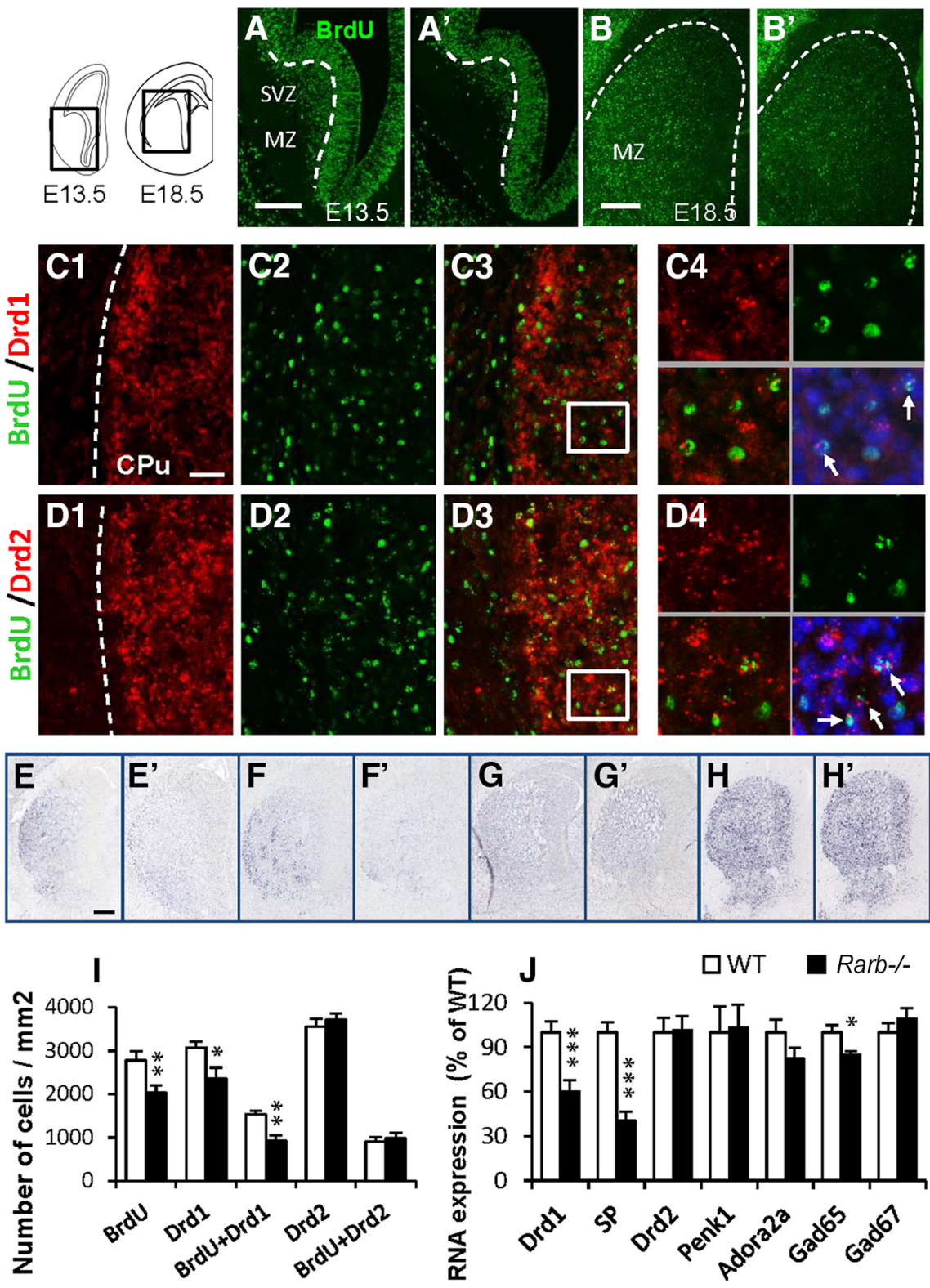

Figure 1. Reduced proliferation of striatonigral neurons in Rarb ${ }^{-1-}$ LGE. A-D4, Coronal brain sections of the LGE or striatum (drawings, top left) were analyzed at E13.5 ( $\boldsymbol{A}$ and $\boldsymbol{A}^{\prime}$ ), E18.5 ( $\boldsymbol{B}$ and $\boldsymbol{B}^{\prime}$ ), and P0 (C1-C4 and D1-D4), following

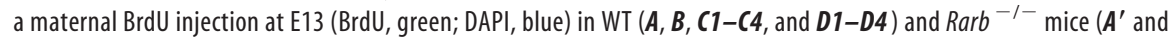
$\left.B^{\prime}\right)$. Drd1-expressing cells (C1-C4, red) and Drd2-expressing cells (D1-D4, red) were identified by fluorescent ISH and their overlap with BrdU immunolabeling is illustrated for WT dorsal striatum at P0 (C2-C4 and D2-D4). C4, D4, I, Details of colabeled cells are indicated by arrows at higher magnification for $\operatorname{Drd1}(\mathbf{C} 4)$ and $\operatorname{Drd} 2(\mathbf{D} 4)$, whereas respective cell counts are shown in $\boldsymbol{I}$. $\boldsymbol{E}-\boldsymbol{H}^{\prime}$, Expression of $\operatorname{Drd} 1\left(\boldsymbol{E}\right.$ and $\left.\boldsymbol{E}^{\prime}\right), \operatorname{Pdyn}\left(\boldsymbol{F}\right.$ and $\left.\boldsymbol{F}^{\prime}\right), C h m r 4\left(\boldsymbol{G}\right.$ and $\left.\boldsymbol{G}^{\prime}\right)$ and $\operatorname{Drd} 2\left(\boldsymbol{H}\right.$ and $\left.\boldsymbol{H}^{\prime}\right)$ were tested at E18.5 and are shown for WT $(\boldsymbol{E}, \boldsymbol{F}, \boldsymbol{G}, \boldsymbol{H})$ and $\operatorname{Rarb}^{-/-}\left(\boldsymbol{E}^{\prime}, \boldsymbol{F}^{\prime}, \boldsymbol{G}^{\prime}\right.$, and $\left.\boldsymbol{H}^{\prime}\right)$ striatum. $\boldsymbol{J}$, mRNA expression of striatonigral (Drd1, SP) and striatopallidal markers (Drd2, Penk, Adora2a) was quantified by qRT-PCR in E18.5 LGE preparations. Scale bars: $\boldsymbol{A}, 500 \mu \mathrm{m} ; \boldsymbol{B}, 200 \mu \mathrm{m} ; \boldsymbol{C} 1,50 \mu \mathrm{m} ; \boldsymbol{E}, 200 \mu \mathrm{m} .{ }^{*} p<0.05,{ }^{* *} p<0.01$, ${ }^{* * *} p<0.001$ compared with WT group (Student's $t$ test). Error bars represent SEM.

septum, and were absent from the LGE (both in WT and Rarb ${ }^{-1-}$ mice) as determined by ISH and qRT-PCR. Expression of Fgf3 was detected in the SVZ of the WT LGE, but was reduced in the SVZ of Rarb $^{-1-}$ embryos (Fig. $2 F, F^{\prime}, I$ ). Reduced expression of Fgf3 is likely to be of transcriptional origin, as Fgf3 is one of the direct RA transcriptional targets (Murakami et al., 1993). Compromised Fgf3 expression may result in reduced global FGF signaling in the SVZ of the Rarb $^{-l-}$ LGE, since other Fgfs (FGF1, FGF2, FGF4, and FGF5) acting as ligands of FGF receptors (FGFR1 and FGFR2) implicated in 

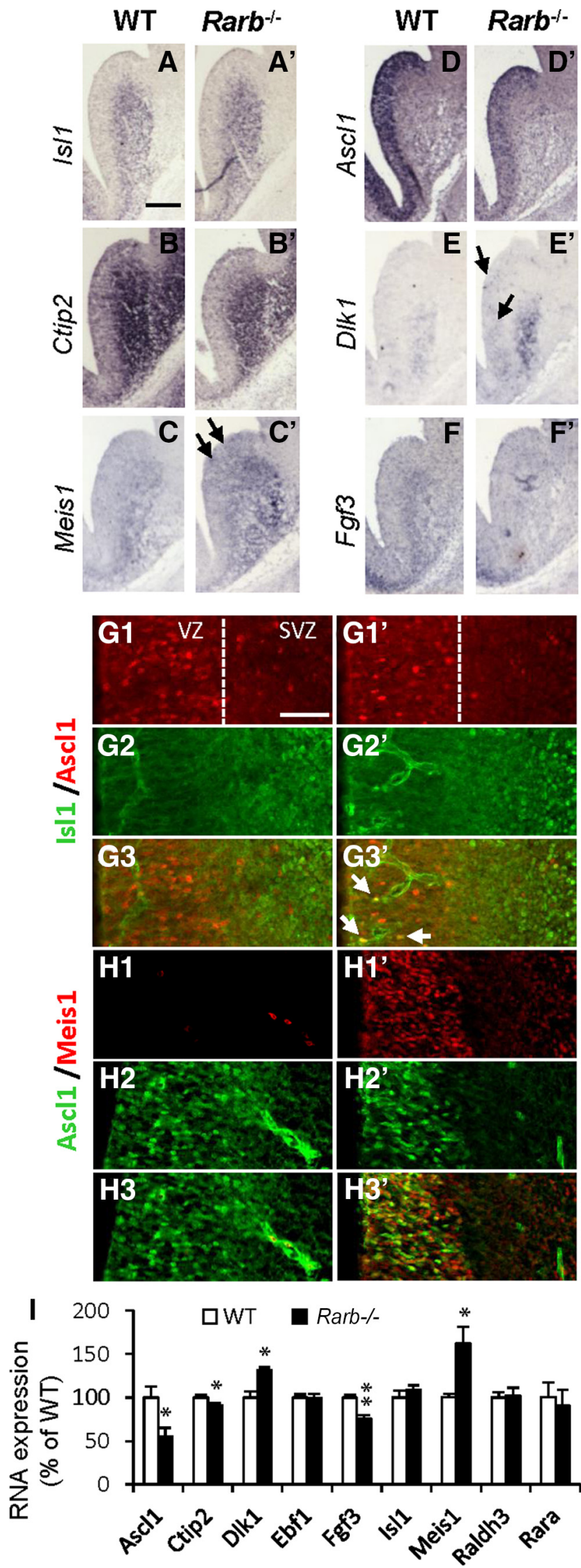

Figure 2. RAR $\beta$ ablation selectively increases differentiation markers at the expense of determinants of neural progenitors in the E13.5 LGE. $\boldsymbol{A}-\boldsymbol{F}^{\prime}$, ISH analyses were performed with various gene markers (as detailed on left side of panels), and are illustrated on coronal sections
A
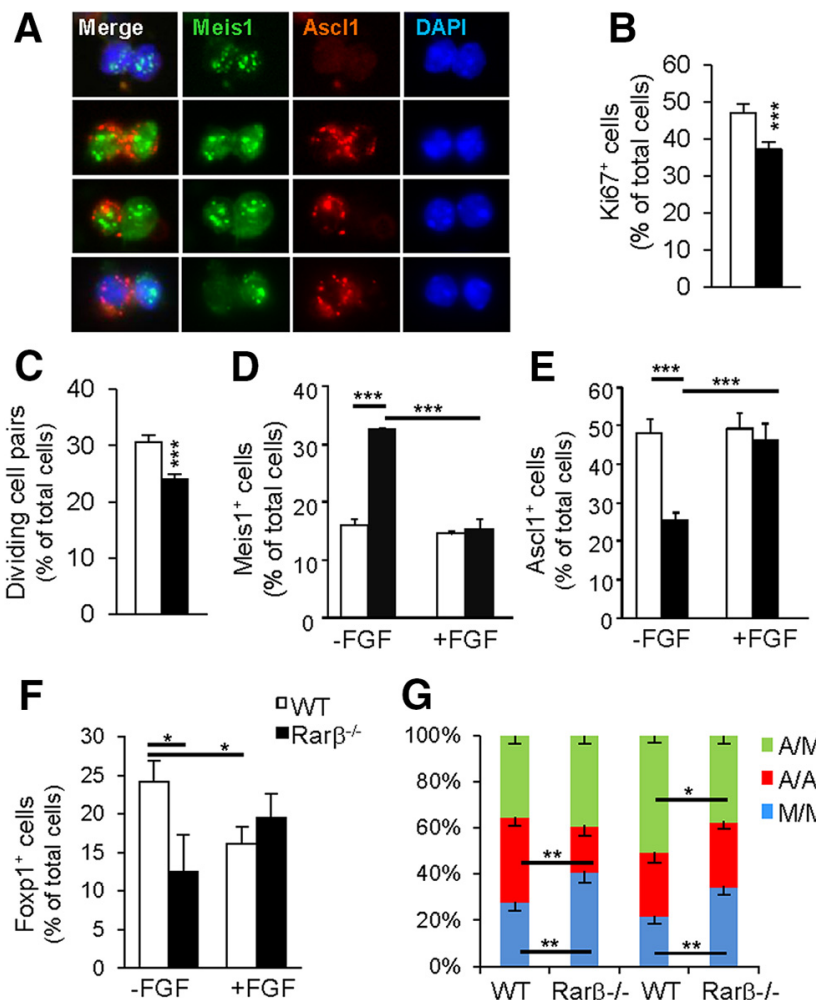

G

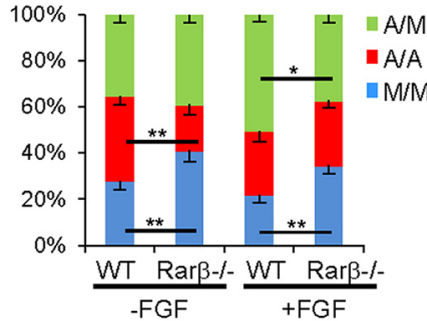

Figure 3. Cell-pair analysis of progenitor cells in Rarb ${ }^{-/-}$LGE primary cell cultures. $\boldsymbol{A}$ Examples of Meis1 and Ascl1 detection in cell pairs from LGE primary cell cultures are shown, illustrating symmetric (top row: Meis1 +/Ascl1 - ; second row: Meis1 +/Ascl1+), and asymmetric (bottom rows) distributions of molecular determinants. $B, C$, The proliferative status of WT versus Rarb ${ }^{-1-}$ LGE cells was investigated by scoring Ki67-positive cells, and number of cell pairs (both expressed as percentage of total DAPI+ cells). $\boldsymbol{D}-\boldsymbol{F}$, 0verall numbers of Meis1+, Ascl1+, and Foxp $1+$ cells (regardless of cell pairs) were also scored after culture in neurobasal $(-\mathrm{FGF}$ ) and in trophic (+FGF) conditions (percentages of DAPI-labeled cells). G, Distribution of cell pairs reflecting symmetric differentiating division (M/M: Meis1+/Ascl1 - cell pairs; $A$, top row), symmetric "neurogenic" division (A/A: Ascl1 + /Ascl1 + cell pairs; $A$, second row), and asymmetric division (A/M: Meis1+/Ascl1 - cell present in one daughter cell; $A$, two bottom rows). ${ }^{* * *} p<0.001$ compared with WT or as indicated on the graph (Student's $t$ test). Error bars represent SEM.

LGE development (Gutin et al., 2006) were not detected in the E13.5 LGE as determined by qRT-PCR and ISH. A consequence of FGF deficiency may be increased differentiation of neural progenitor cells in the VZ and SVZ.

To better understand the dependence among FGF, Meis1, and Ascll signaling in regulation of neuronal differentiation, we performed a series of ex vivo analyses in primary LGE cell cultures, using molecular markers of neuronal differentiation combined with cell-pair analyses (Fig. 3A) as described previously (Shen et al., 2004; Tucker et al., 2010). Similarly to in vivo conditions,

of the anterior region of the LGE. Black arrows indicate ectopic expression domains. G1-G3', Immunofluorescence detection of Isl1 (green) and Ascl1 (red) is shown on coronal sections throughout VZ and SVZ regions (dotted line) of the anterior LGE of WT (G1, G2, and G3) and Rarb $^{-1-}\left(\mathbf{G} \mathbf{1}^{\prime}, \mathbf{G} \mathbf{2}^{\prime}\right.$, and $\left.\mathbf{G} \mathbf{3}^{\prime}\right)$ embryos. (ells coexpressing both markers are indicated by arrows. H1-H3', Immunofluorescence detection of Ascl1 (green) and Meis1 (red) is shown in the anterior LGE of WT $(\boldsymbol{H} \mathbf{1}, \mathbf{H} \mathbf{2}$, and $\boldsymbol{H} \mathbf{3})$ and $\operatorname{Rarb}^{-1-}\left(\boldsymbol{H} \mathbf{1}^{\prime}, \mathbf{H 2}^{\prime}\right.$, and $\left.\boldsymbol{H} \mathbf{3}^{\prime}\right)$ embryos. I, mRNA expression of selected developmental markers in WT and Rarb ${ }^{-1-}$ E13.5 LGE was standardized with respect to $36 B 4$ housekeeping gene and shown as percentage of WT expression levels. Scale bars: $A, 500 \mu \mathrm{m} ; \mathbf{G 1}, 50 \mu \mathrm{m} .{ }^{*} p<0.05,{ }^{* *} p<0.01,{ }^{* * *} p<0.001$ compared with WT group (Student's $t$ test). Error bars represent SEM. 
proliferative deficits were reproduced in a $24 \mathrm{~h}$ cell culture of dissociated LGE cells from E13.5 Rarb $^{-1-}$ embryos seeded at clonal density. Indeed, the percentage of proliferating cells identified by Ki67 immunolabeling was reduced by $23 \%$ in cultures established from $\mathrm{Rarb}^{-1-}$ LGE, compared with WT LGE (Fig. $3 B$ ). Such deficits were further confirmed by a $22 \%$ reduction in the overall numbers of cell pairs in $\mathrm{Rarb}^{-1-}$ cultures, presumably corresponding to cells that divided after plating in vitro (Fig. $3 C$ ). Importantly, the percentage of cells immunolabeled for Meis1 was significantly increased, whereas the percentage of cells expressing Ascl1 (Mash1) was significantly decreased in Rarb ${ }^{-1-}$ cultures (Fig. 3D,E, - FGF histogram), further supporting the possibility of premature differentiation in the E13.5 Rarb $^{-1-}$ LGE. In contrast to increased Meis1, the number of Foxp1-positive cells was reduced in Rarb ${ }^{-1-}$ LGE cultures, thus reproducing reduced Foxp 1 expression observed by qRT-PCR in Rarb $^{-1-}$ LGE (Fig. 3F). In presence of FGF, this difference was normalized mainly through decreased number of Foxp1-positive cells in WT but not in Rarb ${ }^{-1-}$ LGE cultures.

To assess whether altered neuronal differentiation in $\mathrm{Rarb}^{-1-}$ LGE cultures involves cell-extrinsic factors, such as deficient trophic milieu due to reduced Fgf3 expression, we cultured dissociated LGEs from E13.5 embryos in B27-supplemented medium in the presence of FGF2 (bFGF), an easily accessible isotype of FGF, but with similar activity to FGF3. We found that FGF2 treatment normalized the imbalance between Meis1-expressing and Ascl1-expressing cells, as this treatment significantly reduced the number of Meis1-expressing cells (Fig. 3D, compare -FGF, +FGF histograms), while increasing the Ascl1-positive cells (Fig. 3E, compare -FGF, +FGF histograms), in the $\mathrm{Rarb}^{-1-}$ samples.

Cell-pair analyses give an approximate overview of the percentage of cells undergoing (or which underwent) symmetric versus asymmetric divisions (Shen et al., 2004; Tucker et al., 2010). We studied the expression of Ascll as neurogenic marker and Meis1 as marker of differentiating cells in presumed daughter cells, as judged by proximity (Fig. $3 A$, example of asymmetric division). By seeding dissociated LGE cells at low, clonal density we could easily identify pairs expressing Meis1 (but not Ascl1) in both daughter cells in WT and Rarb ${ }^{-1-}$ cultures maintained in $\mathrm{N} 2$ neurobasal medium. However, the percentage of such pairs was $\sim 30 \%$ higher in Rarb $^{-1-}$ cultures $(28 \pm 4 \%$ of all cell pairs for WT and $50 \pm 5 \%$ for Rarb ${ }^{-1-}, p<0.01$ ), reflecting an increased number of symmetric differentiating divisions (Fig. 3G). In contrast, the percentage of cell pairs expressing Ascl1 in both daughter cells and presumably corresponding to cells that underwent symmetric "neurogenic" division (i.e., which were committed to neurogenic fate but retained a progenitor/proliferative capacity) was significantly reduced in Rarb ${ }^{-1-}$ cultures $(37 \pm 3 \%$ in WT, $20 \pm 4 \%$ in $\left.\mathrm{Rarb}^{-1-} ; p<0.01\right)$. Importantly, addition of FGF increased the percentage of Ascl1+/Ascl1+ cell pairs in $\mathrm{Rarb}^{-1-}$ cultures, leading to a normalization of the deficit (28 \pm $4 \%$ in WT, $28 \pm 3 \%$ in Rarb $^{-1-}$; not significant), with a concomitant reduction of Meis1+/Meis1+ cell pairs in both WT and $\mathrm{Rarb}^{-1-}$ cultures, although the percentage of these cell pairs still remained higher in $\mathrm{Rarb}^{-1-}$ samples $(22 \pm 4 \%$ in WT, $34 \pm 4 \%$ in $\left.\mathrm{Rarb}^{-1-} ; p<0.01\right)$. Interestingly, cell pairs with asymmetric expression of Ascl1 (i.e., in only one of the presumed daughter cells) were not affected by genotype in cultures in neurobasal medium ( $35 \pm 3 \%$ in WT, $39 \pm 3 \%$ in Rarb $^{-1-}$; not significant), whereas addition of FGF increased the percentage of these cell pairs in WT cultures, but not in Rarb ${ }^{-1-}$ cultures $(51 \pm 3 \%$ in WT, $37 \pm 4 \%$ in Rarb $^{-1-}$; genotype $\times$ FGF treatment; $F_{(1,12)}=$

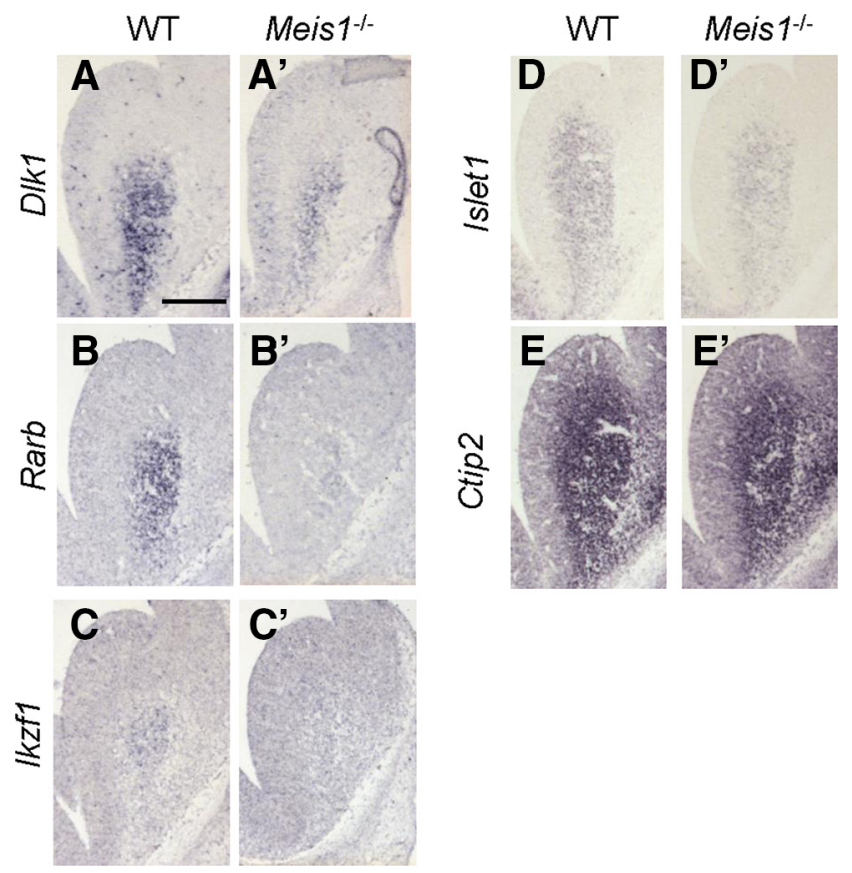

Figure 4. Molecular abnormalities in the Meis $1^{-/-}$LGE at E13.5. $A-E^{\prime}$, ISH was performed with several gene markers (as indicated on left side of panels) on coronal sections of WT $(A, B, C$, $\boldsymbol{D}$, and $\boldsymbol{E})$ and Meis $1^{-1-}\left(\boldsymbol{A}^{\prime}, \boldsymbol{B}^{\prime}, \boldsymbol{C}^{\prime}, \boldsymbol{D}^{\prime}\right.$, and $\left.\boldsymbol{E}^{\prime}\right)$ embryos. The anterior region of the $L G E$ is shown. Scale bar: $A, 500 \mu \mathrm{m}$.

7.8; $p<0.05)$. Thus, an FGF-induced shift promoting symmetric neurogenic divisions (Ascl1+/Ascl1 + ) at the expense of differentiating (Meis1+/Meis + ) cell divisions could possibly explain FGF effects on the global size of Ascl1-expressing and Meis1expressing cell populations in $\mathrm{Rarb}^{-1-}$ LGE cultures.

\section{Analysis of Meis $^{-/-}$embryos supports a role for Meis1 in neuronal differentiation in the developing striatum}

The above data indicate that a gain of Meis 1 function associated with compromised RAR $\beta$ signaling would lead to accelerated neurogenesis in the developing LGE. To further investigate whether Meis1 is required for neuronal differentiation in the LGE, we analyzed Meis $1^{-1-}$ mutant mice (Azcoitia et al., 2005). Several molecular alterations were detected in the developing LGE of Meis $1^{-1-}$ embryos, which may indicate an impaired potential for terminal differentiation. Specifically, expression of $D l k 1$, a transcriptional target of Meis1 and promoter of neuronal differentiation, was strongly decreased throughout the SVZ of the LGE at E13.5 (Fig. $4 A, A^{\prime}$ ). Furthermore, expression of Rarb or Ikzf1, used here as markers of differentiating neurons, were also significantly decreased in the Meis $1^{-1-}$ LGE (Fig. $4 B-C^{\prime}$ ). In contrast, expression of Isl1 and Ctip2, implicated in specification of striatonigral neurons, were less affected in the Meis $1^{-1-}$ LGE (Fig. $4 D-E^{\prime}$ ).

\section{Discussion}

RA is an important modulator of striatal development and controls formation of striosomal compartments (Liao et al., 2008) and differentiation of medium spiny neurons (Chatzi et al., 2011). However, it is not clear how such modulations relate to development of striatonigral and striatopallidal output pathways. Here we demonstrate that RAR $\beta$ is implicated in development of a subpopulation of the striatonigral projection neurons. Accordingly, in addition to reduced levels of Gad65, functionally associated with striatonigral projection neurons (Laprade and 
Soghomonian, 1997), we also observed in Rarb ${ }^{-1-}$ mice a significant reduction of Drd1 and $S P$, some of the key markers specifically enriched in these neurons (Lobo et al., 2006). Such deficits were evident at E16.5 and E18.5, although their severity was stronger at E16.5. At this stage we also observed a reduced expression of Gad67. Recovery of Gad67 expression at E18.5 could suggest delayed neurogenesis of some, but not all, GABAergic neurons. Importantly, compromised neurogenesis observed at E13 did not affect development of striatopallidal projection neurons as expression of Drd2, Penk, or Adora2a were not affected at E16.5 and E18.5. In agreement with our observations at prenatal stages, we found significantly lower number of Drd1-positive neurons, but not of Drd2-positive neurons, in $\mathrm{Rarb}^{-1-}$ striatum at birth. Whereas RAR $\beta$ control of development of Drd1-positive neurons may explain reduced expression of $D r d 1$ in compound Rarb;Rxrg-null mutants (Krezel et al., 1998), the reduced expression of $\operatorname{Drd} 2$ also reported in these animals may involve postnatal functions of RAR $\beta$, which remain to be investigated.

Since we did not observe increased apoptotic cell death at any of the analyzed stages, we investigated whether abnormal proliferation and/or neurogenesis could underlie deficits in striatonigral projection neurons in $\mathrm{Rarb}^{-1-}$ mice. BrdU pulse-chase analyses revealed a significant decrease in neurogenesis in Rarb $^{-/}$LGE at E13, shown previously to affect late-born striosomal neurons (Liao et al., 2008). Although such deficit could affect development of different cell types in the striatum, we focused our analyses on striatonigral and striatopalldial projection neurons. Importantly, we show now that deficits in proliferation are associated with compromised development of striatonigral neurons, because the number of BrdU-positive cells expressing $\operatorname{Drd} 1$ was significantly reduced in the striatum of $\mathrm{Rarb}^{-1-}$ mice at P0, whereas Drd2-expressing BrdU-positive cells were not affected. Reduced expression of prodynorphin, a marker of striatonigral striosomal neurons, and spared expression of Ebfl and Chrm4, respective markers of developing and mature striatonigral matrix neurons, suggest that deficits in Drd1 neurons are restricted to striosomal compartments. Several lines of evidence suggest that reduced proliferation observed in the $\mathrm{Rarb}^{-1-}$ LGE result from premature differentiation of neural progenitors in the VZ and SVZ regions. Deficits in neurogenesis at E13.5 illustrated by reduced expression of Ascl1 (Mash1), a marker of neural progenitors and a determinant of GABAergic neurons in the striatum (Casarosa et al., 1999; Yun et al., 2002), was associated with concomitant increase in expression of Meis1, which we considered as a marker of postmitotic neurons due to its expression in the $\mathrm{MZ}$ of the developing striatum and throughout the adult striatum, which harbor postmitotic neurons. Furthermore, Meis1 was expressed ectopically in the VZ of the Rarb ${ }^{-1-}$ LGE, where it was found essentially in cells not expressing Ascll, suggesting their premature differentiation. The relevance of increased activity of Meis1 was supported by enhanced expression of $D l k 1$, a direct transcriptional target of Meis1 (Argiropoulos et al., 2008), which is known as positive regulator of neurogenesis and terminal differentiation by induction of cell-cycle exit (Surmacz et al., 2012). We also found ectopic or enhanced expression of Isl1 protein in the VZ and SVZ regions. Although signaling through this transcription factor could promote differentiation of striatonigral projection neurons, as evidenced by previous reports (Ehrman et al., 2013; Lu et al., 2014), in Rarb ${ }^{-1-}$ mice its ectopic expression in the VZ was found only occasionally in few isolated cells. Also, enhanced immunofluorescent labeling of Isl1 did not reflect increased expression of Isl1 at the mRNA level, suggesting the possibility of either post-transcriptional control or

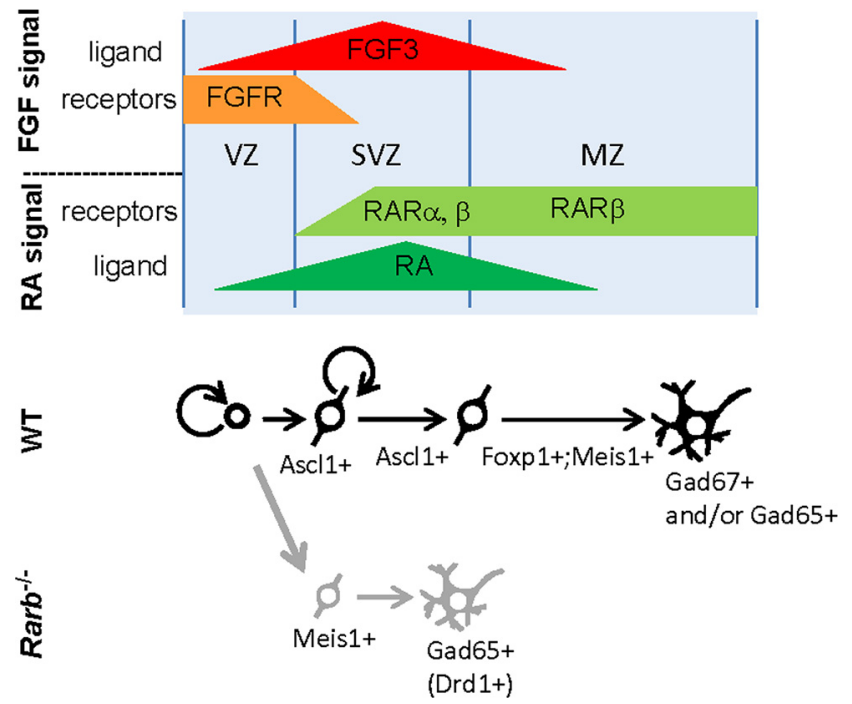

Figure 5. Schematic model of morphogenic gradients controlling striatal development and involved in abnormal neurogenesis in Rarb ${ }^{-1-}$ mice. Top, Developing LGE is represented in blue. A gradient of RA activity, generated through combined ligand (Raldh3dependent) and receptor (RAR $\alpha$ and RAR $\beta$ in SVZ, RAR $\beta$ in MZ) distributions, is presumably highest in the SVZ. By activating Fgf3 expression, this would allow FGF signaling to be transduced by FGF receptors (FGFR) mainly present in the VZ. Bottom, Neurogenesis in WT mice (black arrows) involves progressive neural specification of stem cells (circle) and cell divisions of neural progenitors (crossed circles). In Rarb ${ }^{-1-}$ mice, a subpopulation of progenitor cells prematurely expresses Meis 1 in the VZ and SVZ regions, leading to premature differentiation of striatonigral neurons and thus impoverishment of the pool of striatonigral progenitors that normally undergo differentiation through a Foxp1-dependent and Ikzf1-dependent pathway.

relatively subtle or local changes in Isl1 expression. Also, we did not observe any mis-specification of Drd1 to Drd2 neurons, which could be expected in case of overexpression of Isl1 as previously suggested (Lu et al., 2014). We observed a slight decrease in expression of Ctip2, a transcription factor important for development of striatonigral projection neurons and cellular architecture of the striatum (Arlotta et al., 2008). Functional relevance and critical contribution of such deficit to abnormal striatal development in $\mathrm{Rarb}^{-1-}$ mice is unlikely because we failed to identify enhanced expression of Secretagogin, Fidgetin, Neurotensin, or Basonuclin 2 at E18.5, the key molecular hallmarks of abolished Ctip2 expression in neonatal striatum (Arlotta et al., 2008).

It was striking that ablation of RAR $\beta$, predominantly expressed in the MZ and weakly in the SVZ (Liao et al., 2003; M. Rataj and W. Krezel, unpublished observations), affects gene expression and cell proliferation in a neighboring region (the VZ) that does not express this receptor. We hypothesized that RAR $\beta$ triggers noncell-autonomous events by regulating the production of extrinsic, diffusible signals, such as RA, or FGFs known for their regulation of proliferation and differentiation of neural progenitors (Chatzi et al., 2011; Guillemot and Zimmer, 2011). We did not observe any signs of altered bioavailability of RA, as expression of Raldh3, the only Raldh gene expressed in the developing LGE, was not affected in Rarb ${ }^{-1-}$ mutants. Also we did not observe any adaptive changes in expression of Rar $\alpha$, known to be expressed in the VZ of the LGE (Rar $\gamma$ was not detected in WT or Rarb $^{-1-}$ LGE). On the other hand, several lines of evidence suggest that reduced expression of $F g f 3$, which we observed in the SVZ region, underlies neurogenesis deficits in the $\mathrm{Rarb}^{-1-}$ LGE. Reduced expression of $\mathrm{Fg} 33$ could be of transcriptional origin, as Fgf3 is a direct transcriptional target of retinoid receptors (Mu- 
rakami et al., 1993; Frenz et al., 2010). While FGF3 and FGF8 act redundantly for specification of the ventral telencephalon at early stages of embryogenesis (Theil et al., 2008), at E13.5 FGF3 is the main FGF available in the proliferative region (VZ and SVZ) of the LGE, no expression being detected in this region for FGF1, FGF2, FGF4, FGF5, and FGF8. FGF3 activity is likely restricted to the VZ by the presence of FGF receptors, in particular FGFR1 and FGFR2, which were demonstrated to play critical roles in development of the LGE since their genetic ablation led to the absence of ventral telencephalic structures (Gutin et al., 2006). In the present study, the deficit in neurogenesis and enhanced differentiation, highlighted, respectively, by the reduced fraction of Ascl1-expressing cells and increased number of Meis1-positive cells, were reproduced in primary cell cultures from E13.5 $\mathrm{Rarb}^{-/-}$LGE, and these changes could be normalized by adding exogenous FGF to the culture medium. Analyses of Ascl1 and Meis1 expression in cell pairs suggest that ablation of RAR $\beta$ leads to increased number of symmetric differentiating divisions identified by expression of Meis1 in both daughter cells at the expense of symmetric neurogenic progenitor $($ Ascl1 +/Ascl1+) cell divisions. FGF treatment essentially normalized this abnormal phenotype (with the exception of a partial decrease of symmetric differentiating divisions) in Rarb $^{-1-}$ LGE, but also enhanced the number of asymmetric divisions in WT but not Rarb $^{-1-}$ cultures, suggesting a critical role of compromised FGF signaling in deficient neurogenesis in $\mathrm{Rarb}^{-1-}$ LGE.

Our data point to the involvement of Meis1 as one of the determinants of differentiation during striatal development. This possibility is further supported by our observation of molecular abnormalities in the LGE of Meis $1^{-/-}$embryos at E13.5. Whether these abnormalities would eventually affect differentiation of striatonigral projection neurons cannot be assessed, as Meis ${ }^{-/-}$ embryos do not survive beyond E14.5 (Azcoitia et al., 2005). Nevertheless, our findings suggest a potential novel mechanism in control of striatonigral neuron differentiation, in addition to pathways involving Isl1 or Ctip2, the key factors reported to play such a role so far (Arlotta et al., 2008; Ehrman et al., 2013; Lu et al., 2014). Such role of Meis1 in brain development may be particularly relevant for the etiology of the restless leg syndrome, for which the MEIS1 locus was recently identified through genomewide association studies and functional analyses (Spieler et al., 2014).

From the present findings, we propose a model of LGE development based on gradients of FGF and RA signaling (Fig. 5). These gradients are imposed not only by diffusion of FGF3 and RA from the SVZ, the main source of both ligands in the LGE, but also by differential distributions of the respective FGF and RA receptors. Thus, FGF3 diffusing from the SVZ will act mostly in the VZ, where FGF receptors are expressed, and therefore will control proliferation of neural progenitor cells, but will also prevent them from terminal differentiation according to a ventrolateral gradient. Cells migrating through the SVZ are exposed to the RA signal produced in the SVZ through Raldh3-mediated synthesis, and perceived by two RARs: $\operatorname{RAR} \alpha$, expressed in the SVZ region, and $\mathrm{RAR} \beta$, found in the $S V Z$ and the only RAR present in the MZ during LGE development. Such exposure to RA in the absence of FGF signal is critical for induction of terminal neuronal differentiation. Molecular changes during the developmental transition from proliferating neural progenitors to postmitotic cells is marked by expression of several transcription factors, including Ikzf1, Foxp1, or Meis1, out of which the first two were reported to control differentiation of MSNs (Tamura et al., 2004; Martín-Ibáñez et al., 2010), whereas the function of Meis1 in this process remains unknown. Our data suggest that Meis1 expression at the entry of a cell into the MZ may be a determinant of terminal differentiation for a subpopulation of striatonigral neurons. FGF signaling might orchestrate this process by preventing Meis1 expression in VZ region. In favor of such negative control are our data from Rarb $^{-/-}$LGE cell cultures. In Rarb $^{-/-}$mutant mice, reduced FGF signaling leads to ectopic expression of Meis1 in VZ cells of the LGE and premature differentiation of a subpopulation of Gad65+ (Drd1+) striatonigral neurons, in turn reducing the pool of progenitor cells that should normally undergo Foxp1-dependent events contributing to differentiation of this pool of projection neurons. Our data from LGE cultures further support this hypothesis as only in WT conditions did FGF decrease the number of Foxp1-positive cells, suggesting a reduction of differentiation, whereas in $\mathrm{Rarb}^{-/-}$cultures the pool of Foxp1positive cells was reduced on expense of Meis1-positive cells, and not affected by FGF treatment. These data suggest that Foxp 1 and Meis1 signaling identify two parallel and mutually exclusive pathways of differentiation, both sensitive to FGF signaling.

\section{References}

Argiropoulos B, Palmqvist L, Yung E, Kuchenbauer F, Heuser M, Sly LM, Wan A, Krystal G, Humphries RK (2008) Linkage of Meis1 leukemogenic activity to multiple downstream effectors including Trib2 and Ccl3. Exp Hematol 36:845-859. CrossRef Medline

Arlotta P, Molyneaux BJ, Jabaudon D, Yoshida Y, Macklis JD (2008) Ctip2 controls the differentiation of medium spiny neurons and the establishment of the cellular architecture of the striatum. J Neurosci 28:622-632. CrossRef Medline

Azcoitia V, Aracil M, Martínez AC, Torres M (2005) The homeodomain protein Meis1 is essential for definitive hematopoiesis and vascular patterning in the mouse embryo. Dev Biol 280:307-320. CrossRef Medline

Casarosa S, Fode C, Guillemot F (1999) Mash1 regulates neurogenesis in the ventral telencephalon. Development 126:525-534. Medline

Chatzi C, Brade T, Duester G (2011) Retinoic acid functions as a key GABAergic differentiation signal in the basal ganglia. PLoS Biol 9:e1000609. CrossRef Medline

Crittenden JR, Graybiel AM (2011) Basal ganglia disorders associated with imbalances in the striatal striosome and matrix compartments. Front Neuroanat 5:59. CrossRef Medline

Diez del Corral R, Olivera-Martinez I, Goriely A, Gale E, Maden M, Storey K (2003) Opposing FGF and retinoid pathways control ventral neural pattern, neuronal differentiation, and segmentation during body axis extension. Neuron 40:65-79. CrossRef Medline

Dollé P, Ruberte E, Leroy P, Morriss-Kay G, Chambon P (1990) Retinoic acid receptors and cellular retinoid binding proteins. I. A systematic study of their differential pattern of transcription during mouse organogenesis. Development 110:1133-1151. Medline

Ehrman LA, Mu X, Waclaw RR, Yoshida Y, Vorhees CV, Klein WH, Campbell $\mathrm{K}$ (2013) The LIM homeobox gene Isll is required for the correct development of the striatonigral pathway in the mouse. Proc Natl Acad Sci U S A 110:E4026-E4035. CrossRef Medline

Erlander MG, Tillakaratne NJ, Feldblum S, Patel N, Tobin AJ (1991) Two genes encode distinct glutamate decarboxylases. Neuron 7:91-100. CrossRef Medline

Frenz DA, Liu W, Cvekl A, Xie Q, Wassef L, Quadro L, Niederreither K, Maconochie M, Shanske A (2010) Retinoid signaling in inner ear development: a "Goldilocks" phenomenon. Am J Med Genet A 152A:29472961. CrossRef Medline

Ghyselinck NB, Dupé V, Dierich A, Messaddeq N, Garnier JM, Rochette-Egly C, Chambon P, Mark M (1997) Role of the retinoic acid receptor beta (RARbeta) during mouse development. Int J Dev Biol 41:425-447. Medline

Guillemot F, Zimmer C (2011) From cradle to grave: the multiple roles of fibroblast growth factors in neural development. Neuron 71:574-588. CrossRef Medline

Gutin G, Fernandes M, Palazzolo L, Paek H, Yu K, Ornitz DM, McConnell SK, Hébert JM (2006) FGF signalling generates ventral telencephalic cells independently of SHH. Development 133:2937-2946. CrossRef Medline 
Krezel W, Ghyselinck N, Samad TA, Dupe V, Kastner P, Borrelli E, Chambon P (1998) Impaired locomotion and dopamine signaling in retinoid receptor mutant mice. Science 279:863-867. Medline

Krzyzosiak A, Szyszka-Niagolov M, Wietrzych M, Gobaille S, Muramatsu S, Krezel W (2010) Retinoid $\times$ receptor gamma control of affective behaviors involves dopaminergic signaling in mice. Neuron 66:908-920. CrossRef Medline

Laprade N, Soghomonian JJ (1997) Glutamate decarboxylase (GAD65) gene expression is increased by dopamine receptor agonists in a subpopulation of rat striatal neurons. Brain Res Mol Brain Res 48:333-345. CrossRef Medline

Liao WL, Chambon P, Liu FC 2003 Retinoic acid receptor-mediated genetic control of pattern formation in the striatum of mammalian telencephalon. Program No. 560.7. Washington, DC: Society for Neuroscience.

Liao WL, Tsai HC, Wang HF, Chang J, Lu KM, Wu HL, Lee YC, Tsai TF, Takahashi H, Wagner M, Ghyselinck NB, Chambon P, Liu FC (2008) Modular patterning of structure and function of the striatum by retinoid receptor signaling. Proc Natl Acad Sci U S A 105:6765-6770. CrossRef Medline

Lobo MK, Karsten SL, Gray M, Geschwind DH, Yang XW (2006) FACSarray profiling of striatal projection neuron subtypes in juvenile and adult mouse brains. Nat Neurosci 9:443-452. CrossRef Medline

Lobo MK, Yeh C, Yang XW (2008) Pivotal role of early B-cell factor 1 in development of striatonigral medium spiny neurons in the matrix compartment. J Neurosci Res 86:2134-2146. CrossRef Medline

Lu KM, Evans SM, Hirano S, Liu FC (2014) Dual role for Islet-1 in promoting striatonigral and repressing striatopallidal genetic programs to specify striatonigral cell identity. Proc Natl Acad Sci U S A 111:E168-E177. CrossRef Medline

Martín-Ibáñez R, Crespo E, Urbán N, Sergent-Tanguy S, Herranz C, Jaumot M, Valiente M, Long JE, Pineda JR, Andreu C, Rubenstein JL, Marín O, Georgopoulos K, Mengod G, Fariñas I, Bachs O, Alberch J, Canals JM (2010) Ikaros-1 couples cell cycle arrest of late striatal precursors with neurogenesis of enkephalinergic neurons. J Comp Neurol 518:329-351. CrossRef Medline

Martynoga B, Morrison H, Price DJ, Mason JO (2005) Foxg1 is required for specification of ventral telencephalon and region-specific regulation of dorsal telencephalic precursor proliferation and apoptosis. Dev Biol 283: 113-127. CrossRef Medline

Molero AE, Gokhan S, Gonzalez S, Feig JL, Alexandre LC, Mehler MF (2009) Impairment of developmental stem cell-mediated striatal neurogenesis and pluripotency genes in a knock-in model of Huntington's disease. Proc Natl Acad Sci U S A 106:21900-21905. CrossRef Medline

Murakami A, Grinberg D, Thurlow J, Dickson C (1993) Identification of positive and negative regulatory elements involved in the retinoic acid/ cAMP induction of Fgf-3 transcription in F9 cells. Nucleic Acids Res 21:5351-5359. CrossRef Medline
Paek H, Gutin G, Hébert JM (2009) FGF signaling is strictly required to maintain early telencephalic precursor cell survival. Development 136: 2457-2465. CrossRef Medline

Ruberte E, Friederich V, Chambon P, Morriss-Kay G (1993) Retinoic acid receptors and cellular retinoid binding proteins. III. Their differential transcript distribution during mouse nervous system development. Development 118:267-282. Medline

Shen Q, Goderie SK, Jin L, Karanth N, Sun Y, Abramova N, Vincent P, Pumiglia K, Temple S (2004) Endothelial cells stimulate self-renewal and expand neurogenesis of neural stem cells. Science 304:1338-1340. CrossRef Medline

Spieler D, Kaffe M, Knauf F, Bessa J, Tena JJ, Giesert F, Schormair B, Tilch E, Lee H, Horsch M, Czamara D, Karbalai N, von Toerne C, Waldenberger M, Gieger C, Lichtner P, Claussnitzer M, Naumann R, Müller-Myhsok B, Torres M, et al. (2014) Restless legs syndrome-associated intronic common variant in Meis1 alters enhancer function in the developing telencephalon. Genome Res 24:592-603. CrossRef Medline

Storm EE, Rubenstein JL, Martin GR (2003) Dosage of Fgf8 determines whether cell survival is positively or negatively regulated in the developing forebrain. Proc Natl Acad Sci U S A 100:1757-1762. CrossRef Medline

Storm EE, Garel S, Borello U, Hebert JM, Martinez S, McConnell SK, Martin GR, Rubenstein JL (2006) Dose-dependent functions of Fgf8 in regulating telencephalic patterning centers. Development 133:1831-1844. CrossRef Medline

Surmacz B, Noisa P, Risner-Janiczek JR, Hui K, Ungless M, Cui W, Li M (2012) DLK1 promotes neurogenesis of human and mouse pluripotent stem cell-derived neural progenitors via modulating Notch and BMP signalling. Stem Cell Rev 8:459-471. CrossRef Medline

Tamura S, Morikawa Y, Iwanishi H, Hisaoka T, Senba E (2004) Foxp1 gene expression in projection neurons of the mouse striatum. Neuroscience 124:261-267. CrossRef Medline

Theil T, Dominguez-Frutos E, Schimmang T (2008) Differential requirements for Fgf3 and Fgf8 during mouse forebrain development. Dev Dyn 237:3417-3423. CrossRef Medline

Tucker ES, Lehtinen MK, Maynard T, Zirlinger M, Dulac C, Rawson N, Pevny L, Lamantia AS (2010) Proliferative and transcriptional identity of distinct classes of neural precursors in the mammalian olfactory epithelium. Development 137:2471-2481. CrossRef Medline

van der Kooy D, Fishell G (1987) Neuronal birthdate underlies the development of striatal compartments. Brain Res 401:155-161. CrossRef Medline

Yun K, Fischman S, Johnson J, Hrabe de Angelis M, Weinmaster G, Rubenstein JL (2002) Modulation of the notch signaling by Mash1 and Dlx1/2 regulates sequential specification and differentiation of progenitor cell types in the subcortical telencephalon. Development 129:5029-5040. Medline 